\title{
Polypeptide-GalNAc-T6 expression predicts better overall survival in patients with colon cancer
}

\author{
LUIS UBILLOS $^{1,2}$, EDGARDO BERRIEL ${ }^{3,4}$, DANIEL MAZAL $^{5}$, SABINA VICTORIA ${ }^{6}$, \\ ENRIQUE BARRIOS $^{7}$, EDUARDO OSINAGA $^{1,4}$ and NORA BEROIS ${ }^{4}$
}

\author{
${ }^{1}$ Departamento de Inmunobiología, Facultad de Medicina, Universidad de la República, \\ Montevideo $11800 ;{ }^{2}$ Servicio de Oncología Clínica, Hospital de Clínicas, Facultad de Medicina, Universidad de la \\ República, Montevideo 11600; ${ }^{3}$ Clínica Quirúrgica 1, Hospital Pasteur, Facultad de Medicina, Universidad de la \\ República; ${ }^{4}$ Laboratorio de Glicobiología e Inmunología Tumoral, Institut Pasteur de Montevideo, Montevideo 11400 ; \\ ${ }^{5}$ Cátedra de Anatomía Patológica, Hospital de Clínicas, Facultad de Medicina, \\ Universidad de la República, Montevideo $11600 ;{ }^{6}$ Unidad de Biología Celular, Institut Pasteur de Montevideo, \\ Montevideo 11400; ${ }^{7}$ Departamento de Métodos Cuantitativos, Facultad de Medicina, Universidad de la República, \\ Montevideo 11800, Uruguay
}

Received September 9, 2017; Accepted April 23, 2018

DOI: $10.3892 / \mathrm{ol} .2018 .8686$

\begin{abstract}
Colorectal carcinoma (CRC) is the second leading cause of cancer mortality worldwide. $O$-glycosylated mucins at the cell surface of colonic mucosa exhibit alterations in cancer and are involved in fundamental biological processes, including invasion and metastasis. Certain members of the GalNAc-transferase family may be responsible for these changes and are being investigated as novel biomarkers of cancer. In the present study the prognostic significance of GalNAc-T6 was investigated in patients with CRC patients. GalNAc-T6 expression was observed in all three colon cancer cell lines analyzed by reverse transcription-polymerase chain reaction, immunofluorescence and flow cytometry. A cohort of 81 colon cancer specimens was analyzed by immunohistochemical staining using MAb T6.3. It was demonstrated that GalNAc-T6 was expressed in 35/81 (43\%) cases of colon cancer but not in the normal colonic mucosa. No association was observed with the clinical-pathologic parameters. However, patients expressing GalNAc-T6 had a significantly increased overall survival (median, 58 months; $\mathrm{P}<0.001$ ) compared with GalNAc-T6 negative patients, especially those with advanced disease. These results suggest that GalNAc-T6 expression predicts an improved outcome in patients with CRC. The molecular mechanism underlying the less aggressive behavior
\end{abstract}

Correspondence to: Dr Nora Berois, Laboratorio de Glicobiología e Inmunología Tumoral, Institut Pasteur de Montevideo, 2020 Mataojo, Montevideo 11400, Uruguay

E-mail: nberois@pasteur.edu.uy

Key words: GalNAc-T6, colon cancer, O-glycosylation, tumor marker of colon cancer cells expressing GalNAc-T6 remains to be elucidated.

\section{Introduction}

Colorectal carcinoma (CRC) is the most common of the gastrointestinal malignancies, and the second leading cause of cancer death in most countries. Colon cancer tissues commonly produce secreted and cell surface-bound mucins (1), which are glycoproteins carrying large numbers of $O$-linked oligosaccharides, accounting for up to $80 \%$ of the molecular mass. Alteration in $O$-glycan profiles is a hallmark of cancer development, which determines the expression of truncated $O$-glycosylated tumor-associated antigens $(2,3)$. These glycoproteins are involved in fundamental biological processes, such as invasion and metastasis (4) as well as in the epithelial-mesenchymal transition (EMT) process (5). Mucins of human colon cancer cells commonly express several types of short $O$-glycan antigens, that can be identified using monoclonal antibodies, but not detected in normal colorectal cells (6). The most extensively characterized are Tn, STn and T antigens $(7,8)$, but the expression by colon cancer tissues of others unusual truncated $O$-glycans, such the Tk antigen (9) and the core6 structure (10) was also reported. Cancer-associated mucin antigens can be exploited in diagnosis and prognosis (3), and for the development of cancer vaccines $(11,12)$. The synthesis of $O$-linked glycosylation is started in the Golgi apparatus by the covalent linkage of an $\alpha-\mathrm{N}$-acetylgalactosamine residue (GalNAc) to the hydroxyl group of Ser/Thr residues. This reaction is catalyzed by the family of UDP-N-acetyl-D-galactosamine:polypeptide $\mathrm{N}$-acetylgalactosaminyltransferase (GalNAc-Ts, EC 2.4.2.41) composed at least by 20 members in humans (13). Following the synthesis of GalNAc $\alpha-O$-Ser/Thr (Tn antigen) other sugar residues are incorporated. In total, 8 mucin-type core structures can be distinguished, depending on the second 
sugar and its sugar linkage, in a process controlled by specific glycosyltransferases (14).

The colon mucus layer is formed by polymerized mucins, primarily MUC2, that are produced by goblet cells (15). Unlike core 1 -derived $O$-glycans that are present in most tissues (16), core 3 -derived $O$-glycans are expressed predominantly in normal colonic epithelial cells (17). Several evidences support the concept that normal mucins and $O$-glycans are key components in colon tissues and that defects in their expression may be associated with an increase in the susceptibility for inflammatory diseases and cancer development $(18,19)$. For example: i) The loss of core 1 -derived $O$-glycans led to a rapid induction of severe spontaneous colitis in mice by 2 weeks after birth (20); ii) the absence of membrane-bound mucin Muc1 leads to the exacerbation of chronic inflammations in both Th1-mediated and Th2-mediated colitis models (21); iii) Muc2-deficient mice (Muc2-/-) developed adenomas at 6 months of age, which progressed to invasive adenocarcinoma in the small intestine as well as rectal tumors at an older age (22), suggesting that Muc2 play a role in the suppression of intestinal cancer; iv) mice lacking core $3 \beta 1,3-\mathrm{N}$-acetylglucosaminyltransfe rase $(\mathrm{C} 3 \mathrm{GnT})$, an important enzyme for the synthesis of core 3 -derived $O$-glycans, exhibited an increased susceptibility to experimental colitis and colorectal adenocarcinoma (23); v) in addition, it was found that inactivating somatic and germline mutations in the gene encoding for GalNAc-T12 (GALNT12) (a gene highly expressed in the normal colon), are associated with colon cancer development (24).

One of the underlying causes of glycosylation changes in cancer is deregulated expression or localization of glycosyltransferases and associated proteins within the tumor cell. In CRC mutations in several genes of glycosylation pathway has been demonstrated (25). Among glycosyltransferases, GalNAc-Ts have been found to be differentially expressed in malignant tissue compared to normal tissue (26-28). Numerous evidences support the role of some GalNAc-Ts in cancer biology (29). It was found that overexpression of GALNT3 promotes pancreatic cancer cell growth (30). Increasing evidences suggest that these enzymes might be useful tumor markers. For example, GalNAc-T3 expression has been shown to correlate with prognosis in patients with lung $(31,32)$ and gallbladder cancer (33). GalNAc-T5 expression was markedly reduced in gastric cancer tissues compared with non-malignant gastric mucosa and was an independent prognostic marker for the overall survival of gastric cancer patients (34). GALNT14 expression was highly associated with lower recurrence-free survival in non-small cell lung cancer (NSCLC) patients (35). GalNAc-T14 also promotes invasive properties of lung adenocarcinoma cells through Wnt dependent HOXB9 expression (36). We demonstrated that GALNT6 expression in bone marrow samples correlated with poor clinical outcome in lymph node-negative breast cancer patients (37). We have also shown, in a human neuroblastoma model, that GALNT13 was the most strongly up-regulated gene in metastatic neuroblasts compared with primary tumor xenograft (38). In the same study, we demonstrated that GALNT13 expression in bone marrow of neuroblastoma patients at diagnosis was a strong predictor of poor clinical outcome. In contrast we found that the brain specific gene GALNT9 is present in neuroblasts derived from primary tumor but absent in bone marrow metastatic ones. In a cohort of 122 neuroblatoma patients, GALNT9 expression in primary tumor was associated with higher overall survival, independently of the standard risk-stratification covariates (39). In this context GalNAc-Ts are emerging as novel prognostic markers and potential new targets for tumor treatments.

Expression of GalNAc-T1, -T2, -T3, -T4 and -T12 was reported in normal colon tissues $(13,40-42)$. Several observations support an important role of some of these isoenzymes in colon carcinogenesis and tumor behavior. For example, some evidences confer to GalNAc-T12 a protective role against colon cancer development (24). Loss of GalNAc-T3 expression was correlated with a higher metastatic potential in a mouse colon cancer model (43) and, in the same way, GalNAc-T3 expression in human CRC significantly enhanced the likelihood of patient survival (44). GalNAc-T6 expression was found in LS174T and T84 human colon cancer cell lines $(45,46)$ but not in normal human colon cells (47). Our aim in the present work was to evaluate the potential role of this enzyme as an immunohistochemical colon cancer marker. We found that GalNAc-T6 expression in colon cancer is an independent prognostic factor indicating better overall survival.

\section{Materials and methods}

Cell culture. Human colon cancer cell lines HT29 (ATCC $\mathrm{HTB}^{\mathrm{TM}}$ ), SW480 [SW-480] (ATCC CCL228 ${ }^{\mathrm{TM}}$ ) and SW620 [SW620] (ATCC CCL227 ${ }^{\mathrm{TM}}$ ) were cultured in Dulbecco's Modified Eagle's Medium (DMEM) supplemented with $10 \%$ fetal bovine serum (FBS), $2 \mathrm{mM}$ L-glutamine and $1 \mathrm{mM}$ pyruvate. Subculture of cells grown in monolayer was carry out after washing once with PBS, incubating them with $0.53 \mathrm{mM}$ EDTA and $0.05 \%$ trypsin in PBS (Gibco; Thermo Fisher Scientific, Inc., Waltham, MA, USA) for $5 \mathrm{~min}$ at $37^{\circ} \mathrm{C}$. Cell pellets were washed in PBS, resuspended in $1 \mathrm{ml}$ of Tri-Reagent (Sigma-Aldrich; Merck KGaA, Darmstadt, Germany) and stocked at $-80^{\circ} \mathrm{C}$ until use.

Patient information and tumor specimens. Eighty-one formalin fixed paraffin-embedded colon cancer tissues were studied. Twenty-eight primary tumors with histopathological diagnosis of colon cancer (all stages), as well as 10 normal colon tissues from distal or proximal resection margin and 8 adenomas with different degrees of dysplastic lesions were obtained from the Department of Pathology, Maciel Hospital, Montevideo. Approval from the Institutional Ethical Committee at the University de la Republica (Comité de Etica para Proyectos de Investigación, Facultad de Medicina, Universidad de la República) was obtained prior to beginning. All participants provided signed written informed consent previous to enrollment in the study. Furthermore, 53 came from a commercial human colorectal cancer tissue-array (IMH-306; Imgenex Corporation, San Diego, CA, USA).

Reverse transcription-polymerase chain reaction (RT-PCR). Total RNA was extracted from colon cancer cell lines with Tri-Reagent (Sigma-Aldrich; Merck KGaA) according to the manufacturer's instructions. Two $\mu \mathrm{g}$ of total RNA were included for first strand cDNA synthesis by using 200 units of M-MLV reverse transcriptase (Invitrogen; Thermo 
Fisher Scientific, Inc.) in the presence of $2 \mu 110 \mathrm{mM}$ of each deoxynucleotide triphosphate (dNTPs) and $200 \mathrm{ng}$ of random hexamers (Thermo Fisher Scientific, Inc.) in a $20 \mu \mathrm{l}$ total reaction volume. After incubation at $37^{\circ} \mathrm{C}$ for $1 \mathrm{~h}$, the mixture was heated to $70^{\circ} \mathrm{C}$, snap-cooled and stored at $-20^{\circ} \mathrm{C}$. Amplification of a $499 \mathrm{bp}$ fragment of GALNT6 transcripts was performed using the follow specific primers: 5'-TCCAAA TCAGGGCTCCAGAAG-3' and 5'-CACCTGCAGCTGCTT CACGTAC-3' (accession no.: Y08565). The PCR mixture (total reaction volume of $25 \mu \mathrm{l}$ ) includes $1 \mathrm{x}$ enzyme buffer, $2.5 \mathrm{mM} \mathrm{MgCl}_{2}, 200 \mu \mathrm{M}$ dNTPs, $400 \mathrm{nM}$ of each primer and 1 unit of Taq DNA polymerase (Thermo Fisher Scientific, Inc.). Amplification was performed for 35 cycles under the following conditions: $30 \mathrm{sec}$ at $94^{\circ} \mathrm{C}, 30 \mathrm{sec}$ at $60^{\circ} \mathrm{C}$ and $1 \mathrm{~min}$ at $72^{\circ} \mathrm{C}$. Fifteen $\mu \mathrm{l}$ of PCR products were analyzed by electrophoresis on $2 \%$ agarose gels by direct visualization after ethidium bromide staining. In order to verify cDNA quality, a 596 bp fragment of $\beta 2$-microglobulin transcripts was amplified under same conditions using the primers 5'-ATGTCTCGCTCCGTG GCCTTAG-3' and 5'-AAGTTGCCAGCCCTCCTAGAGC-3' (accession no.: AB021288).

Immunofluorescence microscopy. Cells plated on glass coverslips were washed with PBS, fixed in methanol-acetone $50 \%$ for $10 \mathrm{~min}$ and stored $\mathrm{a}-20^{\circ} \mathrm{C}$ until use. Coverslips were then defrosted, rehydrated in PBS, and blocked in $30 \%$ goat serum for $20 \mathrm{~min}$. Anti-GalNAc-T6 MAb T6.3 (27) $(10 \mu \mathrm{g} / \mathrm{ml})$ was incubated for $1 \mathrm{hr}$ at room temperature, followed by three washes for 5 min each in PBS. Secondary antibody Alexa FluorR 488 goat-anti mouse IgG (A11029; Invitrogen; Thermo Fisher Scientific, Inc.) was incubated for $1 \mathrm{~h}$ at room temperature and after three washes, monolayers were counterstained with DAPI $1 \mu \mathrm{g} / \mathrm{ml}$, mounted in PBS-glycerol 50\% and analyzed by epifluorescence microscopy.

Analysis of GalNAc-T6 expression on cancer cell lines by flow cytometry. Cells were fixed-permeabilized (4\% PFA, $1 \%$ FBS, $0.1 \%$ Tween 20) and incubated with anti-GalNAc-T6 (T6.3) monoclonal antibody. The specific binding of primary MAb T6.3 to the cell lines was developed with an anti-mouse polyclonal antibody FITC-conjugated (Sigma-Aldrich; Merck $\mathrm{KGaA}$ ) and further analyzed using a CyAn ${ }^{\mathrm{TM}}$ ADP Flow Cytometer (Beckman Coulter, Inc., Brea, CA, USA) and Summit v4.3 software. For each analysis 10,000 counts, gated on a FSC vs SSC dot plot excluding doublets populations, were recorded. Results were expressed as percentage of FITC positive cells and FITC mean fluorescence intensity (MFI). Data were expressed as the mean $+/$-standard deviation. Statistical analysis was determined using one-way analysis of variance (ANOVA) and consequently the Tukey's Multiple Camparison test using GraphPad Prism Sofware v5.00 Demo (GraphPad Software, Inc., La Jolla, CA, USA).

Immunohistochemistry. Parafin-embedded sections were prepared following standard protocols. Sections were deparaffinized and rehydrated, followed by endogenous peroxidase blocking with $3 \% \mathrm{H}_{2} \mathrm{O}_{2}$ in $\mathrm{PBS}$ for $30 \mathrm{~min}$ at room temperature. After three washes, tissues were blocked with $10 \%$ goat serum, $1 \%$ BSA in PBS for $30 \mathrm{~min}$ at room temperature, followed by first antibody incubation at $4^{\circ} \mathrm{C}$ overnight (MAb T6.3 diluted at $10 \mu \mathrm{g} / \mathrm{ml}$ in PBS, $0.1 \%$ Tween 20, 1\% BSA). For every assay, a negative control was performed omitting MAb T6.3. A well characterized strong positive tumor was added in each experiment in order to ensure reproducibility of technical conditions. After washing with PBS, sections were incubated for $1 \mathrm{~h}$ at room temperature with a polyclonal rabbit anti-mouse IgG peroxidase conjugated (Dako, catalogue no.: P 0161) diluted at 1:100 in PBS. Staining reaction was performed with 3,3-diaminobenzidine (DAB) $0.5 \mathrm{mg} / \mathrm{ml}$ in TBS, $0.3 \%$ hydrogen peroxide for $30 \mathrm{~min}$ at room temperature. Sections were then counterstained in Mayer's hematoxylin, dehydrated in ethanol and xylene, and mounted. The immunostaining frequency for each tumor was scored, as previously described (27), based on a 0-3 scale for staining extension [(0) for negative samples or $<10 \%$ stained tumor tissue; (1) for samples stained between 10 and $39 \%$ of tumor tissue; (2) for tumor tissues stained between 40 and $79 \%$; and (3) for tumors with $80 \%$ or more of stained tumor cells]. Signal intensity was scored as strong (3), moderate (2), weak (1), and null (0). Total immunostaining score was obtained adding up both parameters in a 0-6 scale. Scores were established jointly by four observers (LU, DM, EO, NB) in a penta-head microscope.

Statistical analysis. Statistical analysis for FACS experiments was determined using One-way analysis of variance (ANOVA) followed by Tukey's Multiple Camparison test using GraphPad Prism Sofware v5.00 (GraphPad Software, Inc.). Results are from three independent experiments and $\mathrm{P}<0.05$ was considered to indicate a statistically significant difference. Univariate survival analysis was performed using the Kaplan-Meier method and compared with the log-rank test. The cut-off for GalNAc-T6 expression was established as negative (0-1) and positive (2-6). Hazard ratios and 95\% confidence intervals (95\% CIs) were estimated using univariate or multivariate Cox proportional-hazard models. All statistical tests were two-tailed. Proportional hazard assumption was tested by Schoenfeld's method and plotting $(-\log (-\log \mathrm{S}(\mathrm{t})))$. All statistical calculations were performed using STATA v14 (StataCorp LP, College Station, TX, USA).

\section{Results}

GalNac-T6 expression in colon cancer cell lines. Considering that GalNAc-T6 expression was previously found in the T84 and LS174T human colon cancer cell lines $(45,46)$, we evaluated here other colon cancer cells such as HT29, SW480 and SW620. Using RT-PCR we found the mRNA encoding GalNAc-T6 in all three cell lines (Fig. 1A), and these results were confirmed at protein level by immunofluorescence staining, observing a marked expression of GalNAc-T6 (Fig. 1B). The percentage of GalNAc-T6 positive cells was evaluated by flow cytometry (Fig. 2). We found that most colon cancer cells expressed this enzyme: SW480 (96.1\%), SW620 (96.1\%) and HT-29 (85.4\%). The MFI (mean fluorescence intensity) value of GalNAc-T6/FITC positive cells was comparable among these cell lines: SW480 (75.3), SW620 (69.3) and HT-29 (83.8).

GalNAc-T6 is expressed in colon cancer but not in normal colon. The study included 81 patients (53 men and 28 women) 

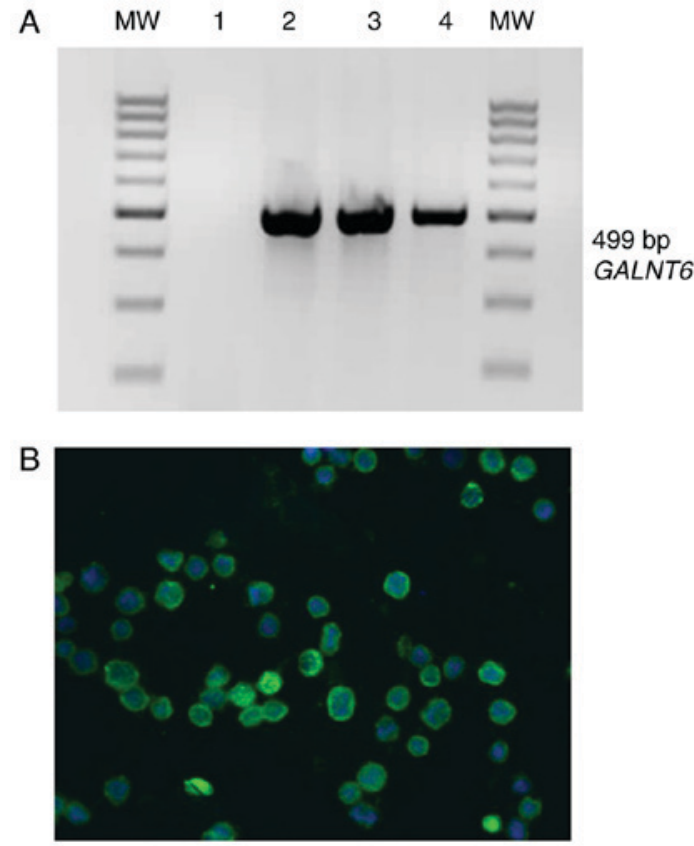

Figure 1. GalNAc-T6 expression in colon cancer cell lines. (A) Reverse transcription-polymerase chain reaction amplifying a $499 \mathrm{bp}$ fragment of GALNT6 messenger. MW, 100 bp molecular weight; 1, negative control (ultrapure water); 2, SW480 cell line; 3, SW620 cell line; 4, HT29 cell line. (B) Immunofluorescence staining of GalNAc-T6 MAb T6.3 in the HT29 cell line. Magnification, x400.

with colorectal cancer diagnosis (Table I). The average age at surgery was 62 years (range 35-86). Most patients (88.8\%) had operable disease at diagnosis and most tumors were graded as well to moderately differentiated type (17.3 and $72.8 \%$ respectively). Based on the American Joint Committee on Cancer (AJCC) criteria, the majority of patients had stage II disease (45.6\%) followed by stage III disease (39.5\%). MAb T6.3 immunostaining was detected in 35 of 81 tumors (43.2\%), in 8/8 specimens from adenomas, and $0 / 10$ normal colon tissues from proximal and distal margin of colonic surgery specimens. This antibody always showed a diffuse cytoplasmic staining pattern with different signal intensity (Figs. 3 and 4). Microwave treatment strongly reduced immunostaining as seen previously for breast cancer (27). No relationship between GalNAc-T6 expression and clinic-pathological factors as age, sex, histology or stage was found (Table I). Regarding disease stage, GalNAc-T6 was expressed in $1 / 3(33.3 \%)$ tumors from patient stage I, in $18 / 37$ $(48.6 \%)$ stage II, in $14 / 32(43.7 \%)$ stage III and in $2 / 9(22.2 \%)$ of stage IV (differences were not statistically significant). No correlation was found between GalNAc-T6 expression and tumor grading, either between tumor staining intensity and the pathological parameter evaluated. Adjacent normal tissue was observed in 16 invasive cancers, and 5 (16\%) showed a focal minimal GalNAc-T6 expression, being all other completely negative (Fig. 4D and F). All ten normal colon tissue obtained from distal or proximal resection margins were negative $0 / 10$ (data not shown).

GalNAc-T6 is an independent prognostic indicator in colorectal cancer. Patients with GalNAc-T6 expression had significantly longer overall postoperative survival (median not reached) compared with those who had GalNAc-T6 negative tumors (median, 58 months, $\mathrm{P}<0.001$; Fig. 5A). In the same way, this benefit was also observed for stage III patients (median survival not reached yet for GalNAc-T6-positive and 36 months for GalNAc-T6-negative, $\mathrm{P}<0.03$ ) and for stage IV, despite the low number of cases, with a median survival of 77 months for GalNAc-T6-positive and 12 months for GalNAc-T6-negative ( $\mathrm{P}<0.02$; Fig. $5 \mathrm{C}$ and $\mathrm{D}$ respectively). No relationship was observed between GalNAc-T6 expression and clinical outcome in stages I and II $(\mathrm{P}=0.63)$. To assess whether GalNAc-T6 expression was an independent predictor of overall postoperative survival, a Cox proportional hazards model was created. Univariate analysis demonstrated that poor tumor differentiation, advanced stage and GalNAc-T6-negative status were significant predictors of poorer survival $(\mathrm{P}=0.028$, 0.007 , and $<0.0001$, respectively). Cox model showed that patients expressing GalNAc-T6 have a hazard ratio of 0.22 $(\mathrm{P}=0.003)$ adjusted by stage, as previously defined. Patients who do not express GalNAc-T6 in their tumors seem to have more than four times higher risk of death compared with those who express it, even considering staging (Table II).

\section{Discussion}

The GALNT6 gene (encoding a type II trans-membrane protein GalNAc-T6), located on chromosome 12q13, is expressed in a restricted pattern, mainly in normal placenta, trachea, brain, pancreas and fibroblast cells (47). Regarding its expression in cancer, GalNAc-T6 level was found significantly higher in breast cancer cells comparing with normal or benign mammary cells (27,48-49). Using a RT-PCR assay, we identified GalNAc-T6 expression in bone marrow samples related to poor clinical outcome in lymph node-negative breast cancer patients (37). It has been demonstrated that high expression of GalNAc-T6 in breast cancer cells correlates with increased glycosylation of the mucin MUC1 and knockdown of GalNAc-T6 suppressed the growth of breast cancer cells (50). On the other hand, $O$-glycosylation of fibronectin (a major constituent of the extracelullar matrix) by overexpressed GalNAc-T6 in breast cancer cell lines causes higher invasiveness related to an EMT-like process (51). In addition, high GalNAc-T6 expression in lung adenocarcinoma was closely related with advanced tumor stage, and independently predicts reduced overall survival of patients (52). In contrast, we observed an opposite significance in colon cancer, for which high GalNAc-T6 expression was correlated with better outcome similar to results reported by Li et al (53), for pancreatic cancer. These apparent contradictory observations could be explained by diverse repertoires of protein acceptor substrates in each tumor type, displaying different biological functions after the incorporation of $O$-GalNAc residues by GalNAc-T6. Similar behavior was reported for GalNAc-T3. High expression of this enzyme correlated with tumor aggressiveness and poor clinical outcome in patients with gallbladder cancer (33), renal cell carcinomas (54) and ovarian cancer (55), while for patients with colon cancer (44), gastric carcinoma (56), and lung adenocarcinoma (32), GalNAc-T3 was a marker of good prognosis. Sometimes this prognosis significance was related to subcellular localization of the enzyme. Miyahara et al (33), reported more intense staining of GalNAc-T3 in gallbladder cancer compared with normal tissue and benign lesions, and 
A

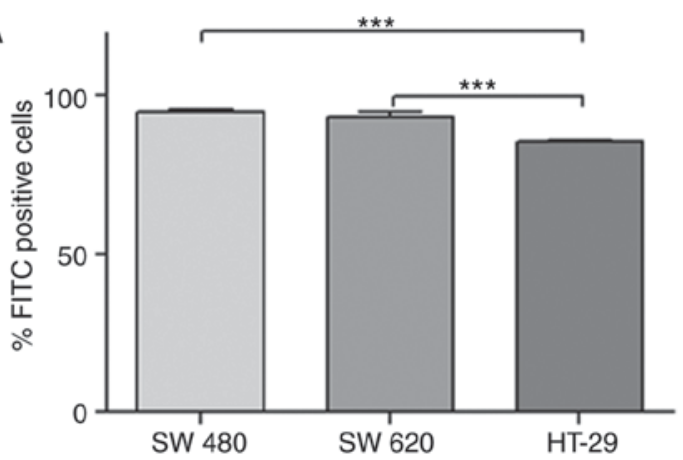

C

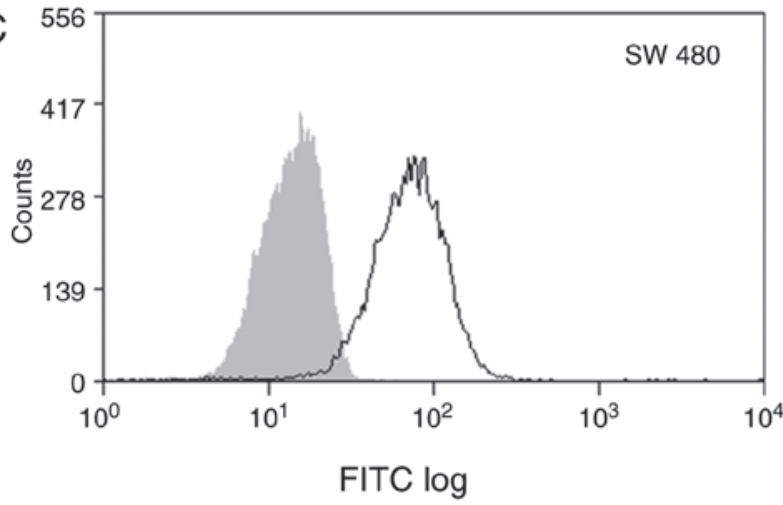

B
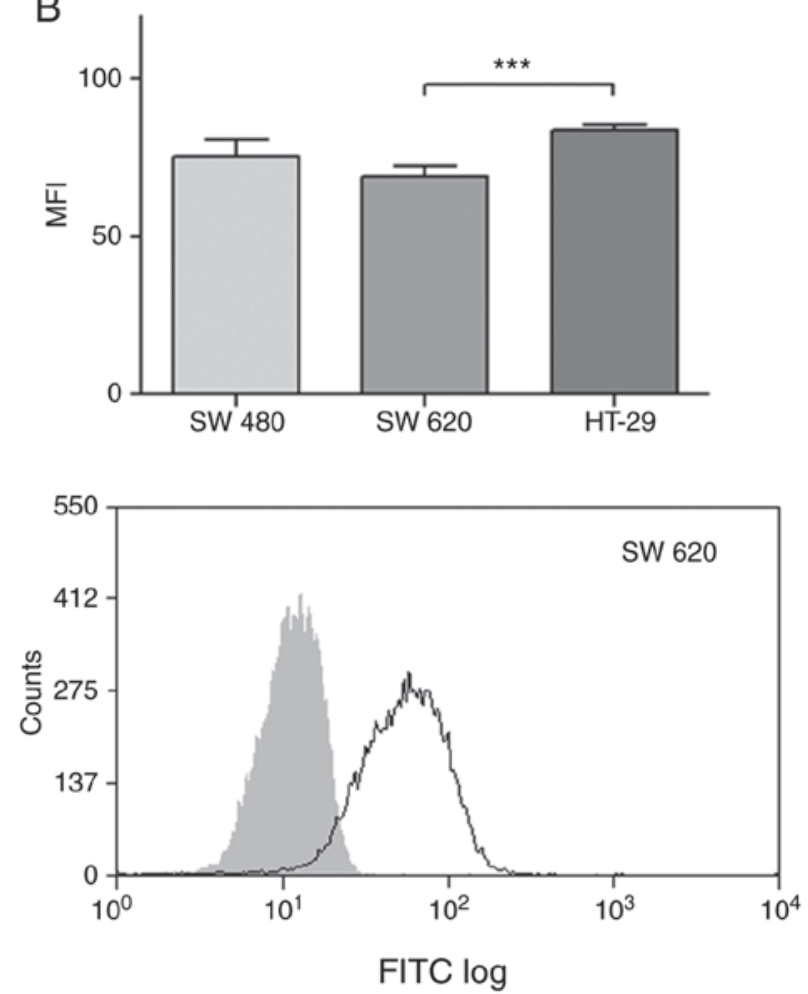

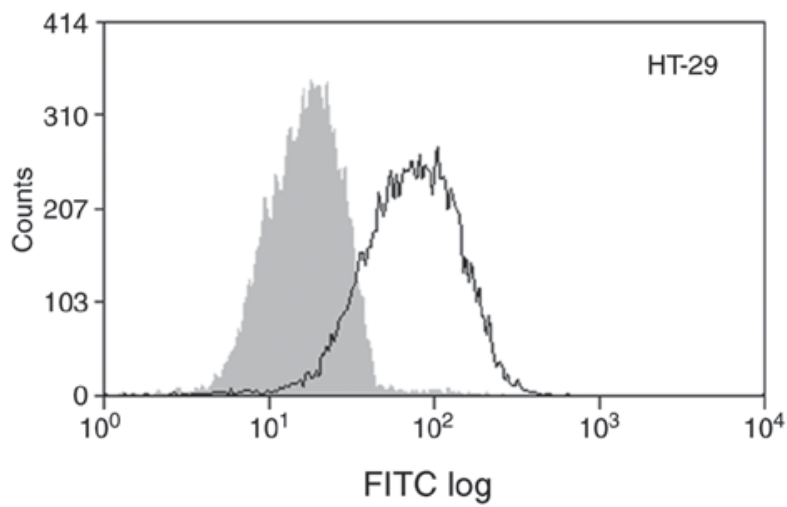

Figure 2. T6.3 MAb recognize native forms of GalNAc-T6 on SW480, SW620 and HT-29 cell lines. GalNAc-T6 expression was evaluated on SW480, SW620 and HT-29 cells, by flow cytometry using a specific MAb and FITC conjugated anti-mouse IgG antibody. (A) The percentage of GalNAc-T6 positive cells; (B) MFI of GalNAc-T6 positive cells (FITC+ cells). (C) Overlay histograms showing the FITC conjugated controls (filled grey) and the GalNAc-T6 expression on the different cell lines (black line). ${ }^{* * *} \mathrm{P} \leq 0.001$. Data are expressed as the mean \pm standard deviation of three independent experiments. MFI, mean fluorescence intensity; FITC, fluorescein isothiocyanate.

also distinguished two well defined staining patterns. While non cancerous tissues always showed a granular perinuclear staining, in gallbladder carcinomas localization was heterogeneous, with granular or diffuse type of subcellular distribution. Importantly, the authors found that postsurgical survival rate of patients with diffuse-type of staining was significantly lower than for patients with granular type. It has been proven that following growth factors stimulation, some GalNAc-Ts (including GalNAc-T6) might be relocalized from the Golgi apparatus to the endoplasmic reticulum (ER), leading to modify $O$-glycosylation of proteins (57).

Precision oncology is becoming increasingly important in CRC management since the great molecular heterogeneity determines large variations in prognosis and response to chemotherapy of individual patients $(58,59)$. Certain biomarkers let to predict clinical outcome beyond staging as well as help in treatment selection (60). The mutational status of $R A S$ and $B R A F \mathrm{~V} 600$ combined with analysis of the DNA mismatch repair system with/without $\mathrm{CpG}$ island methylator phenotype have shown utility to identify colon cancer subtypes with distinct clinical features and prognosis (61). In this work we found that GalNAc-T6 is an independent prognosis biomarker in colon cancer patients. This enzyme was found in the three colon cancer cell lines evaluated (HT-29, SW480 and SW620), both at mRNA and protein level.Immunohistochemical staining of formalin-fixed paraffin embedded tissues using the MAb T6.3 revealed GalNAc-T6 expression in 35/81 (43\%) of cases, without clinical or histological parameters association. We did not observed GalNAc-T6 expression in normal human colon, which is in agreement with results reported by Bennett et al (13) 
Table I. Characteristic of patients and tissues.

\begin{tabular}{lcc}
\hline Characteristics & $\mathrm{n}(\%)$ & GalNAc-T6 positive n (\%) \\
\hline Age, years & & \\
$\quad$ Median (range) & $62(35-86)$ & $18(48.6)$ \\
$\leq 60$ & $37(45.5)$ & $17(38.6)$ \\
$>60$ & $44(54.5)$ & \\
Sex & & $24(45)$ \\
Male & $53(65.5)$ & $11(39)$ \\
Female & $28(34.5)$ & \\
Tumor stage & & $1(33.3)$ \\
I & $3(3.8)$ & $14(48.6)$ \\
II & $37(45.6)$ & $2(22.2)$ \\
III & $32(39.5)$ & \\
IV & $9(11.1)$ & $8(57)$ \\
Histological differentiation & & $26(44)$ \\
Well & $14(17.3)$ & $1(12.5)$ \\
Moderate & $59(72.8)$ & 0.53 \\
Poor & $8(9.9)$ & 0.12 \\
\hline
\end{tabular}
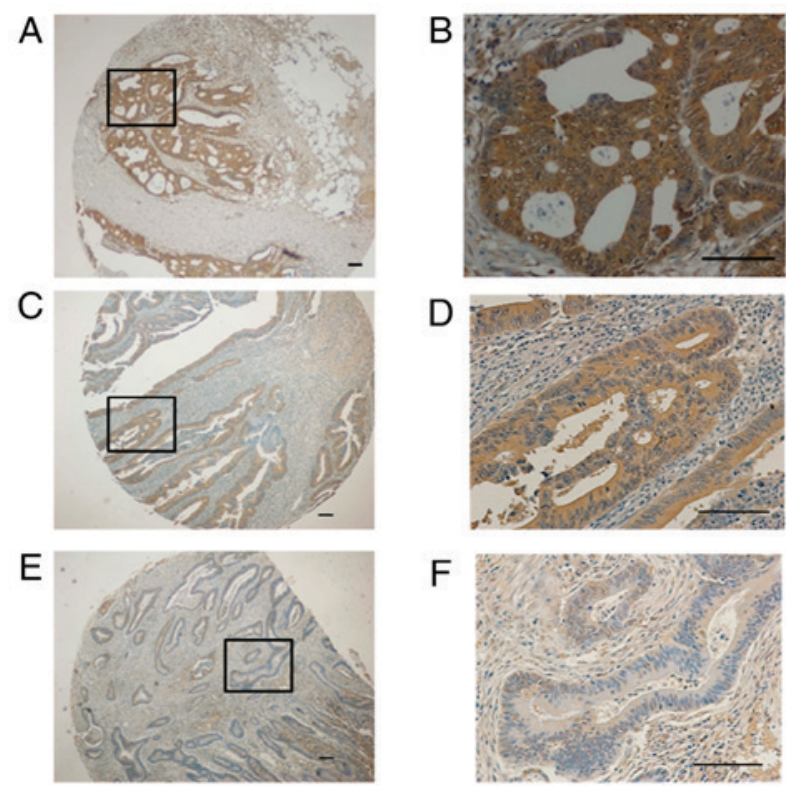

$\mathrm{F}$

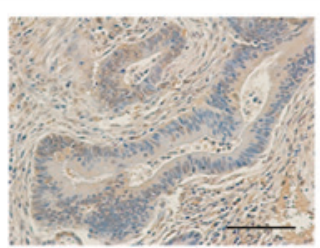

Figure 3. Different signal intensity of GalNAc-T6 expression in colon cancer. (A) Moderately differentiated adenocarcinoma (magnification x40); (B) Detail of figure (A) at magnification, x200 showing a strong stained pattern. (C) Well differentiated adenocarcinoma (magnification, $x 40$ ); (D) detail of figure (C) at magnification, x200 showing moderate stained pattern. (E) Well differentiated adenocarcinoma (magnification $\mathrm{x} 40$ ); (F) detail of figure (E) at magnification x200 illustrating weak stained pattern. All scale bars, $100 \mu \mathrm{m}$.

as well as Lavrsen et al (62), who found GalNAc-T6 expression in colon adenocarcinoma but not in normal colon, using another MAb UH7 (2F3). GalNAc-T6 expression predicts improved overall survival in both, univariate and multivariate analysis. This benefit observed for overall population is maintained in more advance stages (stages III and IV of AJCC), but it was not observed in early stages (I and II of AJCC). A possible
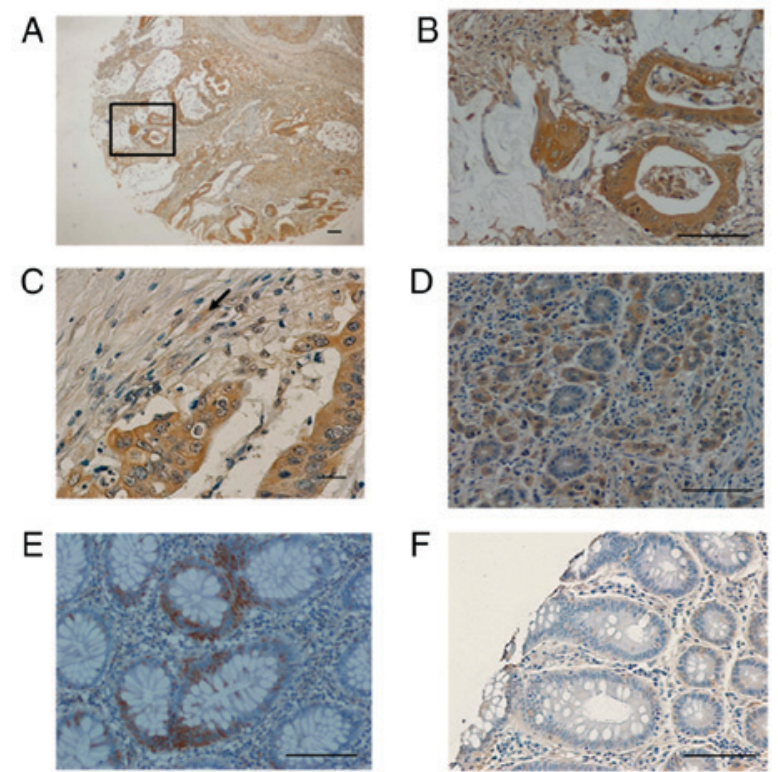

Figure 4. GalNAc-T6 expression in different colon tissues. (A) Mucinous adenocarcinoma (magnification $\mathrm{x} 40$; scale bar, $100 \mu \mathrm{m}$ ); (B) detail of figure (A) at magnifaction x200 (scale bar, $100 \mu \mathrm{m}$ ). (C) Moderatly differentiated colon adenocarcinoma (magnification $\mathrm{x} 400$; scale bar, $20 \mu \mathrm{m}$ ), the arrow indicates GalNAc-T6 expression in fibroblasts as the internal control. (D) Colon adenocarcinoma with moderate staining pattern for GalNAc-T6 expression in tumor cells; normal crypts trapped in tumor tissue are negatives for GalNAc-T6 expression (magnification x200; scale bar, $100 \mu \mathrm{m}$ ). (E) Colon adenoma with strong expression of the enzyme (magnification x200; scale bar, $100 \mu \mathrm{m}$ ). (F) Absence of GalNAc-T6 expression in normal mucosa adjacent to colon cancer (magnification x200; scale bar, $100 \mu \mathrm{m}$ ).

explanation for different clinical impact between pathological stages could be the good prognosis of low stages colorectal cancer itself (I and II) and the higher systemic risk of advanced stages (III and IV). Furthermore, stages III and IV have a confirmed systemic spread (nodal or visceral), and also have 
Table II. Univariate and multivariate analysis.

\begin{tabular}{|c|c|c|c|c|c|c|}
\hline \multirow[b]{2}{*}{ Risk factor } & \multicolumn{3}{|c|}{ Univariate } & \multicolumn{3}{|c|}{ Multivariate } \\
\hline & Hazard ratio & $95 \% \mathrm{CI}$ & P-value & Hazard ratio & $95 \%$ CI & P-value \\
\hline Sex & 1.18 & $0.43-3.16$ & 0.831 & & & \\
\hline Stage & 2.52 & $0.99-6.37$ & 0.007 & 2.74 & $1.11-6.75$ & 0.028 \\
\hline Side & 1.23 & $0.51-2.97$ & 0.470 & & & \\
\hline Histology & 0.31 & $0.02-3.50$ & 0.029 & & & \\
\hline GalNAc-T6 & 0.14 & $0.05-0.38$ & $<0.0001$ & 0.22 & $0.08-0.59$ & 0.003 \\
\hline
\end{tabular}

CI, confidence interval.

A

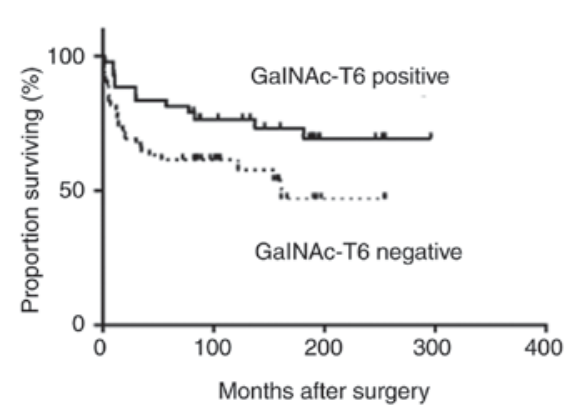

C

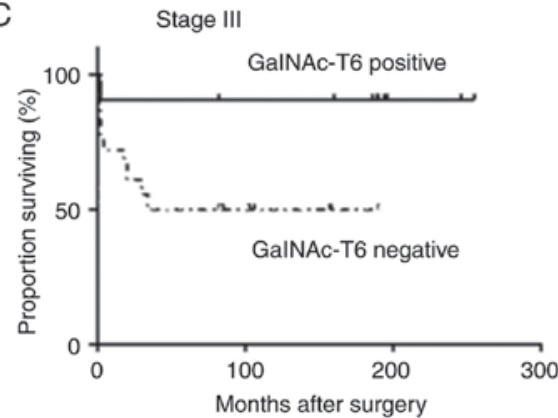

B

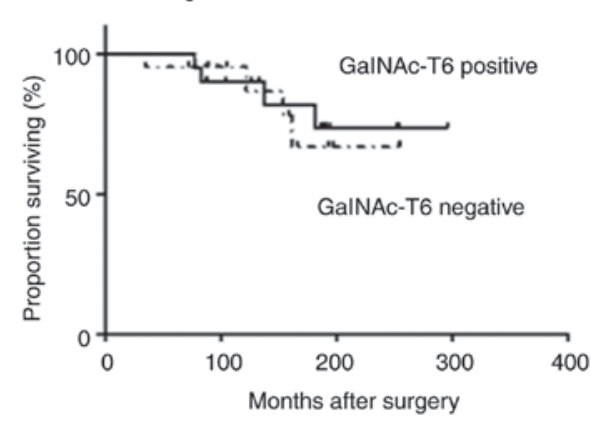

D

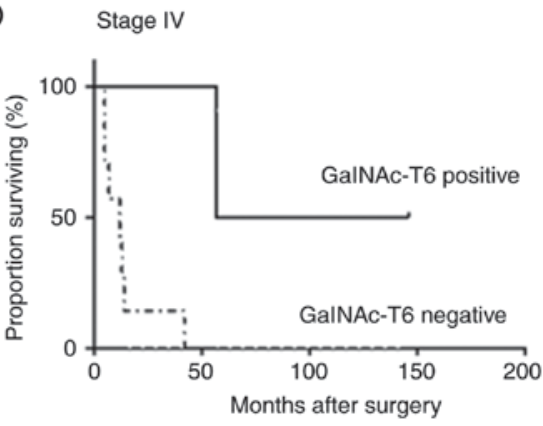

Figure 5. Kaplan-Meier curves for overall survival after surgery according to GalNAc-T6 expression in colorectal cancer patients. (A) All stages, median follow-up 58 months, $\mathrm{P}<0.001$. (B) Stage II patients, $\mathrm{P}=0.63$. (C) Stage III patients, $\mathrm{P}<0.03$. (D) Stage IV patients, $\mathrm{P}<0.02$.

a demonstrated benefit of systemic therapeutic approaches like adjuvant (stage III) or palliative (stage IV) chemotherapy (63), this is also suggested by the null or marginal benefit of adjuvant chemotherapy in stages I and II (64).

The molecular mechanism underlying the less aggressive behavior of colon cancer cells expressing GalNAc-T6 remains to be elucidated. Several studies had revealed the role of GalNAc-Ts in cancer biology, modulating several biological functions as cell adhesion, invasiveness and metastasis (29). GalNAc-T3 and GalNAc-T6 are close paralogs that exhibit very high sequence similarity throughout the coding region, identical genomic structure and encode enzymes with similar substrate specificities $(13,47)$. As we previously exposed, GalNAc-T3 is constitutively expressed in normal colon cells. It is reasonable to think that its glycosylated products could impact in cell-cell and cell-matrix interaction, and its lower expression in colon cancer could affect the way that cancer cell relates with its environment, allowing the cellular escape and metastases. We could hypothesize that if GalNAc-T6 is functional, it could complement the insufficient glycosylation of the lacking GalNAc-T3. This hypothesis is reinforced by the observation that high GalNAc-T3 expression in colon cancer is associated with good clinical outcome (44).

It is too soon to suggest that GalNAc-T6 expression could determine a different treatment in colorectal cancer patients. The retrospective condition of our study, the restricted number of cases, as well as the lack of information about correlation with treatment's response, is main limitation of our work. A prospective trial in a large cohort is needed to confirm its role as prognostic marker. Furthermore the predictive value of response to chemotherapy or biological agents of GalNAc-T6 should be demonstrated prospectively compared with other known markers as $R A S$ and $B R A F$ status. Recently, a molecular classification of colorectal cancer has been proposed: CMS1 (immune), CMS2 (canonical), CMS3 (metabolic) and CMS4 (mesenchymal), 
which could be useful in therapeutic decisions (65). Defining molecular subgroups may identify patients who could benefit from aggressive and targeted therapies, and might be used to select specific treatment approaches for patients with colon cancer. It could be interesting to investigate how GalNAc-T6, other GalNAc-transferases and its products might behave in this molecular classification.

In summary, to the best of our knowledge, this study is the first to prove that GalNAc-T6 expression in colon cancer is an independent predictor for better overall survival, especially in patients with advanced disease (AJCC stages III and IV). It will be necessary to confirm these findings in prospective studies, with larger cohorts, comparing GalNAc-T6 and GalNAc-T3 status and other currently used molecular markers as KRAS, CEA and CA19.9. Additional research is warranted to elucidate the molecular and cellular mechanisms involved in the role of GalNAc-T6 in colon cancer biology.

\section{Acknowledgements}

Not applicable.

\section{Funding}

This work was partially supported by 'Programa Grupos de Investigación' (CSIC, Universidad de la República, Montevideo, Uruguay; grant no.908) and FOCEM (Fondopara la Convergencia Estructural del MERCOSUR; grant no. COF 03-11).

\section{Availability of data and materials}

All data generated or analyzed during this study are included in this published article.

\section{Authors' contributions}

LU performed the experimental work. EBe provided the clinical samples and acquired the data. LU, DM, EO and NB interpreted the data. SV performed the flow cytometry experiments and interpreted the data. EBa performed statistical analysis. EO and NB drafted the manuscript and provided overall supervision of the project. All authors read and approved the final manuscript.

\section{Ethics approval and consent to participate}

The present study was approved by the Institutional Ethical Committee at the University de la Republica and all participants provided written informed consent prior to their enrollment in the present study.

\section{Consent for publication}

All participants provided written informed consent for the publication of their data.

\section{Competing interests}

The authors declare that they have no competing interests.

\section{References}

1. Byrd JC and Bresalier RS: Mucins and mucin binding proteins in colorectal cancer. Cancer Metastasis Rev 23: 77-99, 2004.

2. Dube DH and Bertozzi CR: Glycans in cancer and inflammation-potential for therapeutics and diagnostics. Nat Rev Drug Discov 4: 477-488, 2005.

3. Pinho SS and Reis CA: Glycosylation in cancer: Mechanisms and clinical implications. Nat Rev Cancer 15: 540-555, 2015.

4. Kufe DW: Mucins in cancer: Function, prognosis and therapy. Nat Rev Cancer 9: 874-885, 2009.

5. Freire-de-Lima L, Gelfenbeyn K, Ding Y, Mandel U, Clausen H, Handa K and Hakomori SI: Involvement of O-glycosylation defining oncofetal fibronectin in epithelial-mesenchymal transition process. Proc Natl Acad Sci USA 108: 17690-17695. 2001.

6. Brockhausen I: Mucin-type O-glycans in human colon and breast cancer: Glycodynamics and functions. EMBO Rep 7: 599-604, 2006.

7. Itzkowitz SH, Yuan M, Montgomery CK, Kjeldsen T, Takahashi HK, Bigbee WL and Kim YS: Expression of Tn, sialosyl-Tn, and $\mathrm{T}$ antigens in human colon cancer. Cancer Res 49: 197-204, 1989.

8. Fu C, Zhao H, Wang Y, Cai H, Xiao Y, Zeng Y and Chen H: Tumor-associated antigens: Tn antigen, sTn antigen, and T antigen. HLA 88: 275-286, 2016.

9. Meichenin M, Rocher J, Galanina O, Bovin N, Nifantev N, Sherman A, Cassagnau E, Heymann MF, Bara J, Fraser RH and Le Pendu J: Tk, a new colon tumor-associated antigen resulting from altered O-glycosylation. Cancer Res 60: 5499-5507, 2000.

10. Medina M, Vélez D, Asenjo JA, Egea G, Real FX, Gil J and Subiza JL: Human colon adenocarcinomas express a MUC1-associated novel carbohydrate epitope on core mucin glycans defined by a monoclonal antibody (A10) raised against murine Ehrlich tumor cells. Cancer Res 59: 1061-1070, 1999.

11. Tarp MA and Clausen H: Mucin-type O-glycosylation and its potential use in drug and vaccine development. Biochim Biophys Acta 1780: 546-563, 2008.

12. Kimura T, McKolanis JR, Dzubinski LA, Islam K, Potter DM, Salazar AM, Schoen RE and Finn OJ: MUC1 vaccine for individuals with advanced adenoma of the colon: A cancer immunoprevention feasibility study. Cancer Prev Res (Phila) 6: 18-26, 2013.

13. Bennett EP, Mandel U, Clausen H, Gerken TA, Fritz TA and Tabak LA: Control of mucin-type O-glycosylation: A classification of the polypeptide GalNAc-transferase gene family. Glycobiology 22: 736-756, 2012.

14. Van den Steen P, Rudd PM, Dwek RA and Opdenakker G: Concepts and principles of O-linked glycosylation. Crit Rev Biochem Mol Biol 33: 151-208, 1998.

15. Johansson ME, Larsson JM and Hansson GC: The two mucus layers of colon are organized by the MUC2 mucin, whereas the outer layer is a legislator of host-microbial interactions. Proc Natl Acad Sci USA 108 (Suppl 1): S4659-S4665, 2011.

16. Brockhausen I, Schachter H and Stanley P: Chapter 9 O-GalNAc glycans. In: Essentials of Glycobiology, 2nd edition. Varki A, Cummings RD, Esko JD, Freeze HH, Stanley P, Bertozzi CR, Hart GW, Etxler ME (eds.) Cold Spring Harbor, New York, Laboratory Press, 2009.

17. Xia L: Core 3-derived O-glycans are essential for intestinal mucus barrier function. Methods Enzymol 479: 123-141, 2010.

18. Holst S, Wuhrer M and Rombouts Y: Glycosylation characteristics of colorectal cancer. Adv Cancer Res 126: 203-256, 2015.

19. Bergstrom K, Liu X, Zhao Y, Gao N, Wu Q, Song K, Cui Y, Li Y, McDaniel JM, McGee S, et al: Defective intestinal mucin-type $\mathrm{O}$-glycosylation causes spontaneous colitis-associated cancer in mice. Gastroenterology 151: 152-164.e11, 2016.

20. Fu J, Wei B, Wen T, Johansson ME, Liu X, Bradford E, Thomsson KA, McGee S, Mansour L, Tong M, et al: Loss of intestinal core 1-derived O-glycans causes spontaneous colitis in mice. J Clin Invest 121: 1657-1666, 2011.

21. Nishida A, Lau CW, Zhang M, Andoh A, Shi HN, Mizoguchi E and Mizoguchi A: The membrane-bound mucin Muc1 regulates T helper 17-cell responses and colitis in mice. Gastroenterology 142: 865-874.e2, 2012.

22. Velcich A, Yang W, Heyer J, Fragale A, Nicholas C, Viani S, Kucherlapati R, Lipkin M, Yang K and Augenlicht L: Colorectal cancer in mice genetically deficient in the mucin Muc2. Science 295: 1726-1729, 2002. 
23. An G, Wei B, Xia B, McDaniel JM, Ju T, Cummings RD, Braun $\mathrm{J}$ and $\mathrm{Xia} \mathrm{L}$ : Increased susceptibility to colitis and colorectal tumors in mice lacking core 3-derived O-glycans. J Exp Med 204: 1417-1429, 2007.

24. Guda K, Moinova H, He J, Jamison O, Ravi L, Natale L, Lutterbaugh J, Lawrence E, Lewis $\mathrm{S}$, Willson JK, et al: Inactivating germ-line and somatic mutations in polypeptide $\mathrm{N}$-acetylgalactosaminyltransferase 12 in human colon cancers. Proc Natl Acad Sci USA 106: 12921-12925, 2009.

25. Venkitachalam S and Guda K: Altered glycosyltransferases in colorectal cancer. Expert Rev Gastroenterol Hepatol 11: 5-7, 2017.

26. Mandel U, Hassan H, Therkildsen MH, Rygaard J, Jakobsen MH, Juhl BR, Dabelsteen E and Clausen H: Expression of polypeptide GalNAc-transferases in stratified epithelia and squamous cell carcinomas: Immunohistological evaluation using monoclonal antibodies to three members of the GalNAc-transferase family. Glycobiology 9: 43-52, 1999.

27. Berois N, Mazal D, Ubillos L, Trajtenberg F, Nicolas A, Sastre-GarauX,MagdelenatHandOsinagaE:UDP-N-acetyl-D-g alactosamine:polypeptide $\mathrm{N}$-acetylgalactosaminyltransferase- 6 as a new immunohistochemical breast cancer marker. J Histochem Cytochem 54: 317-328, 2006.

28. Wu C, Guo X, Wang W, Wang Y, Shan Y, Zhang B, Song W, Ma S, Ge J, Deng H and Zhu M: N-Acetylgalactosaminyltransferase-14 as a potential biomarker for breast cancer by immunohistochemistry. BMC Cancer 10: 123, 2010.

29. Hussain MR, Hoessli DC and Fang M: N-acetylgalactosaminyltransferases in cancer. Oncotarget 7: 54067-54081, 2016.

30. Taniuchi K, Cerny RL, Tanouchi A, Kohno K, Kotani N, Honke K, Saibara T and Hollingsworth MA: Overexpression of GalNAc-transferase GalNAc-T3 promotes pancreatic cancer cell growth. Oncogene 30: 4843-5484, 2011.

31. Gu C, Oyama T, Osaki T, Li J, Takenoyama M, Izumi H, Sugio K, Kohno K and Yasumoto K: Low expression of polypeptide GalNAc N-acetylgalactosaminyl transferase-3 in lung adenocarcinoma: Impact on poor prognosis and early recurrence. Br J Cancer 90: 436-442, 2004.

32. Zhao S, Guo T, Li J, Uramoto H, Guan H, Deng W and Gu C: Expression and prognostic value of GalNAc-T3 in patients with completely resected small $(\leq 2 \mathrm{~cm})$ peripheral lung adenocarcinoma after IASLC/ATS/ERS classification. Onco Targets Ther 8: 3143-3152, 2015

33. Miyahara N, Shoda J, Kawamoto T, Furukawa M, Ueda T, Todoroki T, Tanaka N, Matsuo K, Yamada $\mathrm{Y}$, Kohno $\mathrm{K}$ and Irimura $\mathrm{T}$ : Expression of UDP-N-acetyl-alpha-D-galactosamine-polypeptide $\mathrm{N}$-acetylgalactosaminyltransferase isozyme 3 in the subserosal layer correlates with postsurgical survival of pathological tumor stage 2 carcinoma of the gallbladder. Clin Cancer Res 10 2090-2099, 2004

34. He H, Shen Z, Zhang H, Wang X, Tang Z, Xu J and Sun Y: Clinical significance of polypeptide $\mathrm{N}$-acetylgalactosaminyl transferase-5 (GalNAc-T5) expression in patients with gastric cancer. Br J Cancer 110: 2021-2029, 2014.

35. Lee ES, Son DS, Kim SH, Lee J, Jo J, Han J, Kim H, Lee HJ, Choi HY, Jung Y, et al: Prediction of recurrence-free survival in postoperative non-small cell lung cancer patients by using an integrated model of clinical information and gene expression. Clin Cancer Res 14: 7397-7404, 2008.

36. Kwon OS, Oh E, Park JR, Lee JS, Bae GY, Koo JH, Kim H, Choi YL, Choi YS, Kim J and Cha HJ: GalNAc-T14 promotes metastasis through Wnt dependent HOXB9 expression in lung adenocarcinoma. Oncotarget 6: 41916-41928, 2015.

37. Freire T, Berois N, Sóñora C, Varangot M, Barrios E and Osinaga E: UDP-N-acetyl-D-galactosamine:polypeptide $\mathrm{N}$-acetylgalactosaminyltransferase 6 (ppGalNAc-T6) mRNA as a potential new marker for detection of bone marrow-disseminated breast cancer cells. Int J Cancer 119: 1383-1388, 2006.

38. Berois N, Blanc E, Ripoche H, Mergui X, Trajtenberg F, Cantais S, Barrois M, Dessen P, Kågedal B, Bénard J, et al: ppGalNAc-T13: A new molecular marker of bone marrow involvement in neuroblastoma. Clin Chem 52: 1701-1172, 2006.

39. Berois N, Gattolliat CH, Barrios E, Capandeguy L, Douc-Rasy S, Valteau-Couanet D, Bénard J and Osinaga E: GALNT9 gene expression is a prognostic marker in neuroblastoma patients. Clin Chem 59: 225-233, 2013.
40. Kohsaki T, Nishimori I, Nakayama H, Miyazaki E, Enzan H, Nomoto M, Hollingsworth MA and Onishi S: Expression of UDP-GalNAc:polypeptide N-acetylgalactosaminyltransferase isozymes $\mathrm{T} 1$ and $\mathrm{T} 2$ in human colorectal cancer. J Gastroenterol 35: 840-848, 2000.

41. Bennett EP, Hassan $\mathrm{H}$ and Clausen $\mathrm{H}$ : cDNA cloning and expression of a novel human UDP-N-acetyl-alpha-D-galactosamine. Polypeptide N-acetylgalactosaminyltransferase, GalNAc-t3. J Biol Chem 271: 17006-17012, 1996.

42. Guo JM, Chen HL, Wang GM, Zhang YK and Narimatsu H: Expression of UDP-GalNAc:polypeptide N-acetylgalactosaminyltransferase-12 in gastric and colonic cancer cell lines and in human colorectal cancer. Oncology 67: 271-276, 2004.

43. Kato K, Takeuchi H, Kanoh A, Miyahara N, Nemoto-Sasaki Y, Morimoto-Tomita M, Matsubara A, Ohashi Y, Waki M, Usami K, et al: Loss of UDP-GalNAc:polypeptide N-acetylgalactosaminyltransferase 3 and reduced $\mathrm{O}$-glycosylation in colon carcinoma cells selected for hepatic metastasis. Glycoconj J 27: 267-276, 2010.

44. Shibao K, Izumi H, Nakayama Y, Ohta R, Nagata N, Nomoto M, Matsuo K, Yamada Y, Kitazato K, Itoh $\mathrm{H}$ and Kohno K: Expression of UDP-N-acetyl-alpha-D-galactosamine-polypeptide galNAc $\mathrm{N}$-acetylgalactosaminyl transferase-3 in relation to differentiation and prognosis in patients with colorectal carcinoma. Cancer 94: 1939-1946, 2002.

45. Kato K, Takeuchi H, Kanoh A, Mandel U, Hassan H, Clausen H and Irimura T: $\mathrm{N}$-acetylgalactosamine incorporation into a peptide containing consecutive threonine residues by UDP-N-acetyl-D-g alactosaminide:polypeptide $\mathrm{N}$-acetylgalactosaminyltransferases. Glycobiology 11: 821-829, 2001.

46. Kanoh A, Takeuchi H, Kato K, Waki M, Usami K and Irimura T: Interleukin-4 induces specific pp-GalNAc-T expression and alterations in mucin O-glycosylation in colonic epithelial cells Biochim Biophys Acta 1780: 577-584, 2008

47. Bennett EP, Hassan H, Mandel U, Hollingsworth MA, Akisawa N, Ikematsu Y, Merkx G, van Kessel AG, Olofsson S and Clausen $\mathrm{H}$ : Cloning and characterization of a close homologue of human UDP-N-acetyl-alpha-D-galactosamine:poly peptide $\mathrm{N}$-acetylgalactosaminyltransferase-T3, designated GalNAc-T6. Evidence for genetic but not functional redundancy. J Biol Chem 274: 25362-15370, 1999.

48. Brooks SA, Carter TM, Bennett EP, Clausen H and Mandel U: Immunolocalisation of members of the polypeptide $\mathrm{N}$-acetylgalactosaminyl transferase (ppGalNAc-T) family is consistent with biologically relevant altered cell surface glycosylation in breast cancer. Acta Histochem 109: 273-284, 2007.

49. Patani N, Jiang W and Mokbel K: Prognostic utility of glycosyltransferase expression in breast cancer. Cancer Genomics Proteomics 5: 333-340, 2008

50. Park JH, Nishidate T, Kijima K, Ohashi T, Takegawa K, Fujikane T, Hirata K, Nakamura Y and Katagiri T: Critical roles of mucin 1 glycosylation by transactivated polypeptide $\mathrm{N}$-acetylgalactosaminyltransferase 6 in mammary carcinogenesis. Cancer Res 70: 2759-2769, 2010.

51. Park JH, Katagiri T, Chung S, Kijima K and Nakamura Y: Polypeptide N-acetylgalactosaminyltransferase 6 disrupts mammary acinar morphogenesis through $\mathrm{O}$-glycosylation of fibronectin. Neoplasia 13: 320-326, 2011.

52. Li Z, Yamada S, Wu Y, Wang KY, Liu YP, Uramoto H, Kohno K and Sasaguri Y: Polypeptide N-acetylgalactosaminyltransferase-6 expression independently predicts poor overall survival in patients with lung adenocarcinoma after curative resection. Oncotarget 7: 54463-54473, 2016.

53. Li Z, Yamada S, Inenaga S, Imamura $\mathrm{T}$, Wu $\mathrm{Y}$, Wang $\mathrm{KY}$, Shimajiri S, Nakano R, Izumi H, Kohno K and Sasaguri Y: Polypeptide $\mathrm{N}$-acetylgalactosaminyltransferase 6 expression in pancreatic cancer is an independent prognostic factor indicating better overall survival. Br J Cancer 104: 1882-1889, 2011.

54. Kitada S, Yamada S, Kuma A, Ouchi S, Tasaki T, Nabeshima A, Noguchi H, Wang KY, Shimajiri S, Nakano R, et al: Polypeptide $\mathrm{N}$-acetylgalactosaminyl transferase 3 independently predicts high-grade tumours and poor prognosis in patients with renal cell carcinomas. Br J Cancer 109: 472-481, 2013.

55. Wang ZQ, Bachvarova M, Morin C, Plante M, Gregoire J, Renaud MC, Sebastianelli A and Bachvarov D: Role of the polypeptide $\mathrm{N}$-acetylgalactosaminyltransferase 3 in ovarian cancer progression: Possible implications in abnormal mucin O-glycosylation. Oncotarget 5: 544-560, 2014. 
56. Onitsuka K, Shibao K, Nakayama Y, Minagawa N, Hirata K, Izumi H, Matsuo K, Nagata N, Kitazato K, Kohno K and Itoh H: Prognostic significance of UDP-N-acetyl-alpha-D-galactosamine: polypeptide $\mathrm{N}$-acetylgalactosaminyltransferase-3 (GalNAc-T3) expression in patients with gastric carcinoma. Cancer Sci 94: 32-36, 2003.

57. Gill DJ, Chia J, Senewiratne J and Bard F: Regulation of O-glycosylation through Golgi-to-ER relocation of initiation enzymes. J Cell Biol 189: 843-858, 2010.

58. Punt CJ, Koopman M and Vermeulen L: From tumour heterogeneity to advances in precision treatment of colorectal cancer. Nat Rev Clin Oncol 14: 235-246, 2017.

59. Mahasneh A, Al-Shaheri F and Jamal E: Molecular biomarkers for an early diagnosis, effective treatment and prognosis of colorectal cancer: Current updates. Exp Mol Pathol 102: 475-483, 2017.

60. Sinicrope FA, Okamoto K, Kasi PM and Kawakami H: Molecular biomarkers in the personalized treatment of colorectal cancer. Clin Gastroenterol Hepatol 14: 651-658, 2016.

61. Dienstmann R, Salazar R and Tabernero J: Personalizing colon cancer adjuvant therapy: Selecting optimal treatments for individual patients. J Clin Oncol 33: 1787-1796, 2015.

62. Lavrsen K, Dabelsteen S, Vakhrushev SY, Levann AMR, Haue AD, Dylander A, Mandel U, Hansen L, Frödin M, Bennett EP and Wandall HH: De novo expression of human polypeptide $\mathrm{N}$-acetylgalactosaminyltransferase 6 (GalNAc-T6) in colon adenocarcinoma inhibits the differentiation of colonic epithelium. J Biol Chem 293: 1298-1314, 2018.
63. Andre T, de Gramont A, Vernerey D, Chibaudel B, Bonnetain F, Tijeras-Raballand A, Scriva A, Hickish T, Tabernero J, Van Laethem JL, et al: Adjuvant fluorouracil, leucovorin, and oxaliplatin in stage II to III colon cancer: Updated 10-year survival and outcomes according to BRAF mutation and mismatch repair status of the MOSAIC study. J Clin Oncol 33: 4176-4187, 2015.

64. Verhoeff SR, van Erning FN, Lemmens VE, de Wilt JH and Pruijt JF: Adjuvant chemotherapy is not associated with improved survival for all high-risk factors in stage II colon cancer. Int J Cancer 139: 187-193, 2016.

65. Guinney J, Dienstmann R, Wang X, de Reyniès A, Schlicker A, Soneson C, Marisa L, Roepman P, Nyamundanda G, Angelino P, et al: The consensus molecular subtypes of colorectal cancer. Nat Med 21: 1350-1356, 2015.

This work is licensed under a Creative Commons Attribution-NonCommercial-NoDerivatives 4.0 International (CC BY-NC-ND 4.0) License. 